\title{
Images of the month: The conundrum of chronic coccidioidomycosis
}

\author{
Authors: Oluwabusola M Bolaji, ${ }^{A}$ Nurul I Zainudin, ${ }^{A}$ Susan Snape, ${ }^{B}$ Gauri Saini ${ }^{C}$ and Vadsala Baskaran ${ }^{D}$
}

KEYWORDS: respiratory infection, histology, imaging

DOI: $10.7861 /$ clinmed.2020-0815

\section{Case presentation}

A 68-year old woman was admitted to a tertiary hospital for an elective removal of a right spheno-orbital tumour. She had a 2-month history of increasing unsteadiness, recurrent falls, right-sided headache, diplopia and increasing confusion. It is noteworthy that she had been on high-dose steroids preoperatively to reduce the significant tumour-associated oedema, predisposing her to an immunocompromised state. There were no intraoperative complications; histology showed grade II atypical meningioma with extensive soft tissue invasion. Postoperatively, the patient desaturated and became tachypnoeic. Computed tomography (CT) pulmonary angiography revealed large bilateral, peripheral cavitating lung lesions and confirmed the presence of a subsegmental pulmonary embolism in the right upper lobe upstream to one of the lesions (Fig 1). The patient underwent bronchoscopy to investigate for possible underlying infective versus malignant pathology. Bronchoalveolar lavage showed evidence of pneumocystis pneumonia (PCP; deoxyribonucleic acid (DNA) positive) and she was treated with a 2-week course of co-trimoxazole. Despite treatment, symptoms of general malaise, dry cough and intermittent confusion ensued with raised inflammatory markers. Due to ongoing concerns of differential diagnoses of vasculitis and malignancy, the patient had a CTguided lung biopsy. Histology and extended culture of the lung biopsy showed features of fungal involvement with associated tissue necrosis and histolytic reaction. Haematoxylin and eosin stain showed multiple large thick-walled spherules consistent with Coccidioides immites (Fig 2). Pan-fungal polymerase chain reaction performed on the tissues rapidly detected Coccidioides

Authors: ${ }^{A}$ foundation year doctor, Nottingham University Hospital NHS Trust, Nottingham, UK; ${ }^{B}$ consultant in microbiology, Nottingham University Hospital NHS Trust, Nottingham, UK; ${ }^{C}$ consultant in respiratory medicine, Nottingham University Hospital NHS Trust, Nottingham, UK; ${ }^{D}$ clinical research fellow in respiratory medicine, Nottingham University Hospital NHS Trust, Nottingham, UK

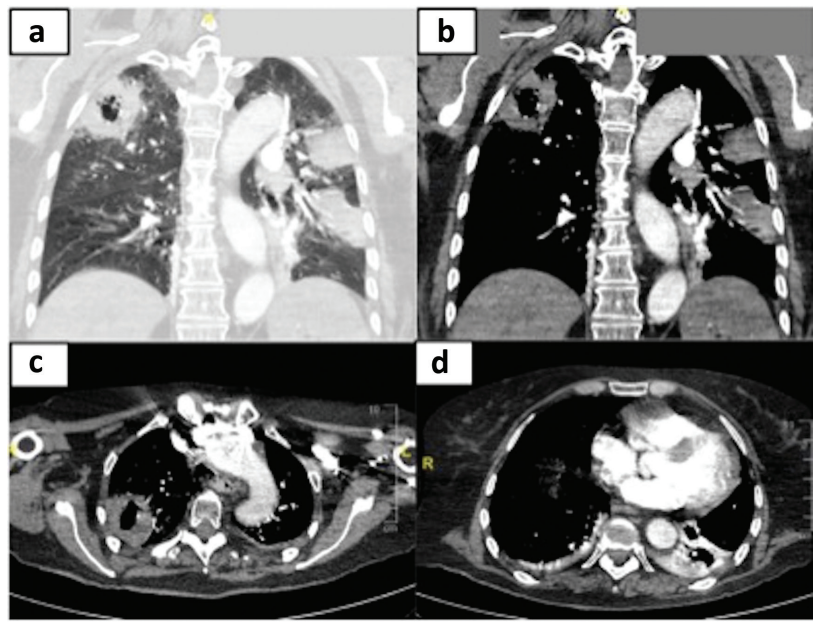

Fig 1. Computed tomography pulmonary angiography showing large bilateral, peripheral cavitating lung lesions with bilateral lower lobe atelectasis, worse on the left. a) Lung window showing coronal planes. b) Mediastinal window showing coronal planes. c) and d) Axial planes.

DNA. Serology tests further confirmed the diagnosis; both Coccidioides mycelial antigen using complement fixation test and Coccidioides immunodiffusion were positive. In light of these findings, upon direct questioning, the patient reported travelling to Phoenix, Arizona, in the USA annually for over 15 years. The patient was diagnosed with chronic coccidioidomycosis, and started on a 1-week course of AmBisome (amphotericin B) before switching to oral fluconazole which will be continued for a year and possibly life-long.

\section{Discussion}

Coccidioidomycosis, also known as valley fever, is a systemic fungal infection caused by $C$ immites or $C$ posadasii. These fungal species are endemic in southwestern USA (particularly Arizona and California), Mexico, Central America and South America. Most infected people are asymptomatic, however, symptomatic individuals present with pulmonary sequelae (selflimiting influenza-like illness or community-acquired pneumonia) as the most common initial syndrome. ${ }^{1}$ As the prevalence of coccidioidomycosis in the UK is entirely due to imported cases 


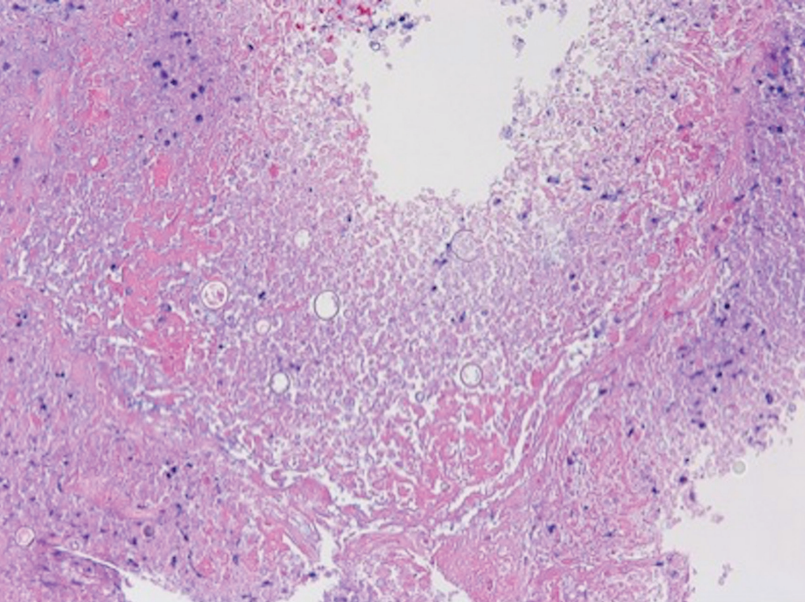

Fig 2. Haematoxylin and eosin stain, from computed tomographyguided lung biopsy, showing multiple large thick-walled spherules consistent with Coccidioides immites.

or laboratory acquisition, suspicion of coccidioidomycosis in our patient was only noted after the provisional histology report showing pathognomonic features of this fungal infection and targeted travel history confirmed the exposure. The indication of whether to treat in complex chronic cases, such as this, is rarely clear. Immunosuppressive medications including corticosteroids have been associated with increased risks of complications from coccidioidal illness. ${ }^{2}$ Therefore, despite having relatively few symptoms, the patient's history of high-dose steroid use, combined with PCP co-infection meant the benefits of therapy outweighed the risks. Although there is a relatively small incidence of the disease in the UK, it is inevitable we will continue to see coccidioidomycosis cases due to the globalisation of travel in the modern world.

\section{References}

1 California Department of Public Health Infectious Diseases Branch. Coccidioidomycosis (valley fever). CDPH, 2018. www.cdph. ca.gov/Programs/CID/DCDC/CDPH \% 20Document \% 20Library/ IDBGuidanceforCALH]s-Cocci.pdf

2 Galgiani JN, Ampel NM, Blair JE et al. 2016 Infectious Diseases Society of America (IDSA) clinical practice guideline for the treatment of coccidioidomycosis. Clin Infect Dis 2016;63:e112-46.

Address for correspondence: Dr Oluwabusola Margaret Bolaji, Nottingham University Hospital NHS Trust, Hucknall Road, Nottingham NG5 1PB, UK.

Email: busolabolaji@hotmail.co.uk 